\title{
Analysis of the Clinical Effects and Expression Levels of Inflammatory Cytokines in Type B AD Patients after TEVAR Therapy
}

\author{
Maimaitiaili Aizezi ${ }^{1}$, Maimaiti Yisireyili ${ }^{2}$, Weimin Zhang ${ }^{1}$, Aziguli Alimujiang ${ }^{2}$, Hongjia Zhang ${ }^{3}$, Lianjun Huang ${ }^{3}$ and Zonggang \\ Zhang ${ }^{1 *}$ \\ ${ }^{1}$ Department of Cardiac Surgery, People's Hospital of Xinjiang Uygur Autonomous Region, Urumqi, Xinjiang, PR. China \\ ${ }^{2}$ Research Institute of General and Minimally Invasive Surgery, People's Hospital of Xinjiang Uygur Autonomous Region, Urumqi, Xinjiang, PR. China \\ ${ }^{3}$ Beijing Aortic Disease Center, Beijing Anzhen Hospital, Capital Medical University, An zhen Road, Beijing, PR. China
}

*Corresponding author: Zonggang Zhang, Department of Cardiac Surgery, People's Hospital of Xinjiang Uygur Autonomous Region, Urumqi, Xinjiang, PR. China, E-mail: zhangzg196219@163.com

Received: 16 Apr, 2019 | Accepted: 07 May, 2019 | Published: 13 May, 2019

Citation: Aizezi M, Yisireyili M, Zhang W, Alimujiang A, Zhang H, et al. (2019) Analysis of the Clinical Effects and Expression Levels of Inflammatory Cytokines in Type B AD Patients after TEVAR Therapy. J Clin Case Stu 4(2): dx.doi.org/10.16966/2471-4925.187

Copyright: (C) 2019 Aizezi M, et al. This is an open-access article distributed under the terms of the Creative Commons Attribution License, which permits unrestricted use, distribution, and reproduction in any medium, provided the original author and source are credited.

\begin{abstract}
Background/Aim: This study aims to investigate the clinical effects and expression levels of inflammatory cytokines in patients with type B acute aortic dissection (AD) after thoracic endovascular aortic repair (TEVAR) therapy.

Methods: A retrospective analysis of 60 patients with acute AD admitted to our hospital from April 2016 to January 2018 was conducted. The patients were divided into control group $(n=30)$ and TEVAR group $(n=30)$ according to different treatment methods administered. The control group was given drug therapy (Urapidil+Felodipine+Diltiazem), and the TEVAR group was given with thoracic endovascular aortic repair therapy. The expression levels of inflammatory cytokines in both groups were measured before (pre) and after (post) the treatment. The therapeutic effects and the long-term outcomes of both groups were analyzed.

Results: The post treatment result shows that the plasma levels of interleukin- 6 (IL-6), interleukin-8 (IL-8), and tumor necrosis factor- $\alpha$ (TNF- $\alpha$ ) were significantly decreased in both groups $(P<0.01)$. Importantly, the plasma levels of IL-6, IL-8, and TNF- $\alpha$ in the TEVAR group were markedly lower than those in the control group $(P<0.01)$. The number of white blood cells $(\mathrm{WBC})$ and neutrophils decreased significantly in the two groups $(P<0.05)$. The number of lymphocytes in the two groups also significantly increased $(P<0.05)$. The numbers of $W B C$, neutrophils, and lymphocytes significantly differed between the TEVAR and control groups $(P<0.05)$. The rate of efficacy in the TEVAR group was significantly higher than that in the control group $(P<0.05)$. The 2 -year survival rate in the TEVAR group was significantly higher than that in the control group $(P<0.05)$. The postoperative complication rate in the TEVAR group was significantly lower than that in the control group $(P<0.05)$.
\end{abstract}

Conclusion: TEVAR exhibits good therapeutic effect by significantly inhibiting the expression of inflammatory cytokines and improving the survival rate to some extent.

Keywords: Acute aortic dissection; Thoracic endovascularaortic repair; Inflammation; Efficacy; Survival rate

\section{Introduction}

Aortic dissection $(\mathrm{AD})$ is the main cause of aortic intimal tear, which induces blood in the aorta to enter the aortic media, thereby rupturing the media and separating the two-chamber aorta [1]. $\mathrm{AD}$ is a relatively dangerous cardiovascular disease (CVD) with rapid progression and high mortality. At present, conservative and surgical treatments are mainly used in clinics. Thoracic endovascular aortic repair (TEVAR) has achieved a major paradigm shift in treatment of atherosclerotic thoracic, aneurysm, and chronic AD [2-3]. Traditionally, resting antihypertensive treatment has been the standard treatment for acute type-B AD.

TEVAR has become the preferred treatment for complex stanford type-B AD. However, differences are still observed on the treatment of non-complex stanford type-B AD. Many researchers advocate the use of TEVAR in treatment of acute $\mathrm{AD}$, whereas some scholars believe that drug therapy should be adopted [4-5]. In this study, we investigated the long-term therapeutic effects of the two treatments and their role in suppressing the expression of inflammatory cytokines.

\section{Methods}

\section{Patients}

A retrospective analysis was conducted on 60 patients with acute AD admitted to the Cardiac Surgery Department of Xinjiang Uygur Autonomous Region People's Hospital from April 2016 to January 2018. The patients were divided into control group $(n=30)$ and TEVAR group $(n=30)$ according to different treatment methods 
administered. Patients in both groups voluntarily participated and signed an informed consent.

\section{Inclusion and exclusion criteria}

The inclusion criteria are as follows: 1) patients who met the diagnostic criteria of acute $\mathrm{AD}[5], 2)$ patients with onset time to treatment time of less than 14 days, and 3) patients subjected to type-B dissection. The exclusion criteria are as follows: 1) patients with traumatic aortic pseudoaneurysm, aortic transection, and other injuries; 2) patients with onset time of more than 14 days of treatment; 3) patients with simple abdominal $\mathrm{AD}$, connective tissue disease, and aortic penetrating ulcer; and 4) patients with systemic consumptive diseases, such as tumors and tuberculosis.

\section{Therapeutic method}

All patients were subjected to electrocardiogram (ECG) monitoring, sedation, and control of blood pressure and heart rate. Radial artery blood pressure was measured and pumped into Uradil (Heilongjiang Fuhe Huaxing Pharmaceutical Group Co., Ltd., Chinese medicine standard word H20040501, specification: $25 \mathrm{mg}$ ). If the effect of blood pressure reduction after 2 hours is not good, then felodipine sustainedrelease tablets are orally administered (Nanjing Yiheng Pharmaceutical Co., Ltd., Chinese medicine standard word H20103396). The systolic blood pressure was maintained at $100-120 \mathrm{mmHg}$, and diltiazem was pumped (Shandong Fangming Pharmaceutical Group Co., Ltd., national medicine standard word H20070254, specification: $10 \mathrm{~mL}: 10$ $\mathrm{mg}$ ) to control the heart rate at 60-70 times per minute.

TEVAR group: CT scanning was conducted to evaluate the condition of dissection, cerebral blood supply, anchorage area, and true or false lumen, determine the location of the entry, understand the type and specifications of the covered stent, and establish the appropriate surgical approach. After strict disinfection, the patients were placed in a horizontal position. A stent was placed in the target position after general anesthesia and tracheal intubation. After marking the position, angiography was performed again at the position of the stent to detect the presence of internal leakage and the artery and incision were sutured. The incision and blood circulation of the operative limb were carefully observed after the operation.

\section{Indication of TEVAR therapy}

Acute phase: 1 . Dissection ruption and bleeding; 2. Progressive enlargement of peri-aortic or mediastinal hematoma; 3. Rapid enlargement of aortic diameter; 4. Severe ischemia of important branches of aorta; 5 . Uncontrollable pain.

Chronic phase: 1 . Dissection ruption and bleeding; 2. Dissection aorta diameter increased rapidly ( $>10 \mathrm{~mm} /$ year); 3. Formation of aneurysms $(>50-60 \mathrm{~mm}) ; 4$. Severe ischemia of important aortic branches (Malperfusion).

\section{Sample collection}

A total of $2 \mathrm{~mL}$ of fasting venous peripheral blood was collected 1 day before and 7 days after the treatment. Serum was collected after centrifugation $\left(15 \mathrm{~min}, 15000 \times \mathrm{g}, 4^{\circ} \mathrm{C}\right)$, and stored at $-80^{\circ} \mathrm{C}$ until use by following the protocols mentioned in previous works [6].

\section{ELISA assays}

Plasma levels of IL-6, IL-8, and TNF- $\alpha$ were measured by ELISA method before and after the treatment. The kits were purchased from R\&D Systems, Minneapolis, MN. The numbers of white blood cells (WBC), neutrophils, lymphocytes, and monocytes were also measured by using Beckmann Kurt Uni Cel DxH 800 five-class hematology analyzer (purchased from Beckmann Kurt Commercial China Co., Ltd).

\section{Evaluation of therapeutic efficacy}

The evaluation criteria of curative effect included the following: the recovery of $\mathrm{AD}$ rupture is significant, the partial recovery of $\mathrm{AD}$ rupture is effective, and the failure of $\mathrm{AD}$ is ineffective. Total effective rate was calculated by (markedly effective+effective) cases/total cases $\times 100$, and the in-hospital mortality rate is (in-hospital) cases/total cases $\times 100$. The complications mainly include hemorrhage, renal insufficiency, infection, and cardiovascular events. The in-hospital incidence is evaluated by complications/total cases $\times 100 \%$. In-hospital curative effect was compared with the total effective rate, in-hospital mortality, in-hospital time, and in-hospital complication rate of both groups.

\section{Follow-up}

Hospital survivors were clinically followed up with letters, emails, and phone calls and by the local referring physician when needed. Computed tomographic angiography (CTA) was performed before discharging all patients. After discharge, every patient was requested to repeat CTA at 1 month, 6 months, and annually after the primary TEVAR. Comparison of long-term and medium-term efficacy: 1-year and 2-year survival rates, incidence of complications after discharge, and secondary intervention rates were compared between the two groups. The complications after discharge mainly included internal leakage, distal recurrence of dissection, and progressive type-A layer. The incidence of complications after discharge is equal to the number of complications after discharge/the total number of complications $\times 100$. The secondary intervention rate is equal to the number of secondary intervention cases/the total number of cases $\times 100$. 1-year survival rate $=1$-year survival cases/total cases $\times 100 \%$; 2 -year survival rate $=2$-year survival cases/total cases $\times 100$.

\section{Statistical analysis}

All data were analyzed using IBM SPSS Statistics for Windows, Version 19.0 (IBM Corp., Armonk, NY, USA). Data were expressed as mean \pm SD. Categorical data were described as number and percentage. Student's t-test was performed to measure the differences between the two groups. Fisher's protected least significant differences test of ANOVA was also performed to analyze the quantitative data collected from both groups. Long-term survival was calculated using KaplanMeier method. A p value of $<0.05$ was used to denote significance.

\section{Results}

\section{TEVAR suppressed the expression levels of inflammatory cytokines}

The pre treatment analysis shows that there was no significant difference in the levels of IL-6, IL- 8 , and TNF- $\alpha$ in both groups $(\mathrm{t}=0.205$, $0.194,0.217, \mathrm{P}>0.05)$. Post treatment result indicates that the levels of IL-6, IL-8 and TNF- $\alpha$ in both groups were significantly lower than those pre treatment $(\mathrm{t}=32.486,22.451,17.645$, 45.612, 33.496, 47.813, $\mathrm{P}<0.01)$. The levels of IL-6, IL- 8 , and TNF- $\alpha$ in the TEVAR group were significantly lower than those in the control group $(t=22.618,18.746$, $21.315, \mathrm{P}<0.01)$. The levels of IL-6, IL-8, and TNF- $\alpha$ in TEVAR group post treatment was markedly lower than control group post treatment $(\mathrm{t}=36.362,30.457,33.652, \mathrm{P}<0.01)$ (Figure 1).

\section{TEVAR decreased the expression levels in immune cells}

The pre treatment analysis of shows that there was no significant difference in the number of WBC, neutrophils, lymphocytes, and 

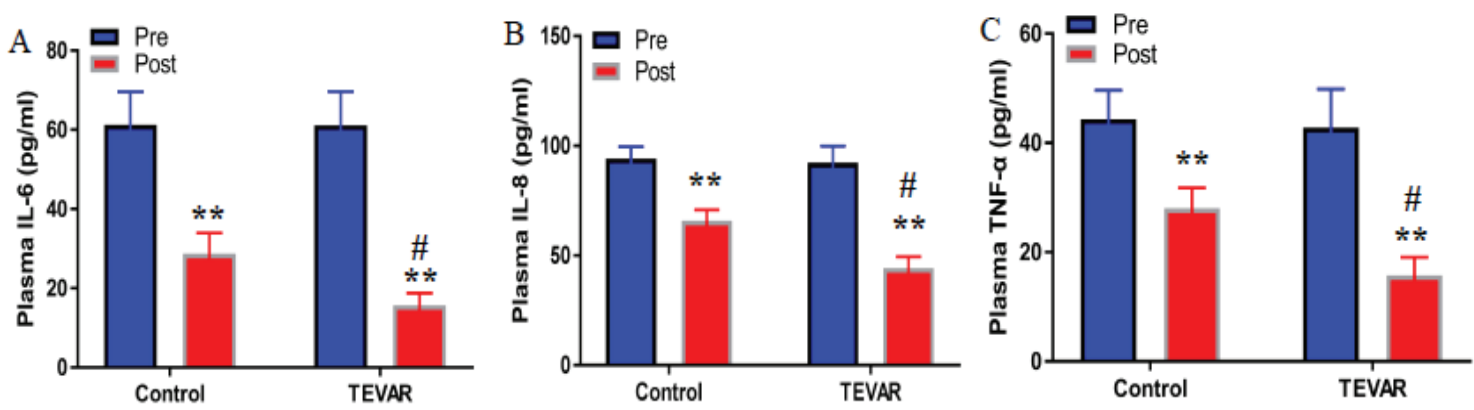

Figure 1: TEVAR suppressed expression levels of inflammatory cytokines.

ELISA was performed to detect the plasma concentrations of IL-6 (A), IL-8 (B), and TNF- $\alpha$ (C). Data are expressed as mean \pm SD ( $n=30$ ). Fisher's protected least significant difference test was performed to analyze the differences between the control and TEVAR groups. ${ }^{* *} \mathrm{P}<0.01$ compared with that pre treatment; $\# \mathrm{P}<0.01$ compared with that post treatment.

monocytes in both groups $(\mathrm{t}=0.187,0.126,0.249,0.423, \mathrm{P}>0.05)$. The post treatment result reveals that the number of WBC and neutrophils in both groups significantly decreased $(\mathrm{t}=5.784,8.312,9.015,8.843$, $\mathrm{P}<0.05$ ), whereas the number of lymphocytes significantly increased $(\mathrm{t}=7.215,9.142, \mathrm{P}<0.05)$. The number of leukocytes and neutrophils in the TEVAR group was significantly lower than that in the control group $(\mathrm{t}=4.568,3.216, \mathrm{P}<0.05)$, and the number of lymphocytes in the TEVAR group was significantly higher than that in the control group $(t=3.856, P<0.05)$. No significant difference in the number of monocytes between the two groups was observed in post treatment $(\mathrm{P}>0.05)$. Furthermore, there was no significant difference in the number of WBC, neutrophils, lymphocytes, and monocytes between TEVAR group post treatment and control group post treatment $(\mathrm{t}=0.128,0.105,0.172,0.087, \mathrm{P}>0.05)$ (Figure 2).

\section{Comparison of the curative effect of both groups of patients}

No significant difference in the basic characteristics of gender and age was observed in both groups $(\mathrm{P}>0.05)$ (Table 1$)$. The post treatment result shows that the effective rate of the TEVAR group was significantly higher than that of the control group $(\mathrm{P}<0.05)$. No significant difference in the mortality, length of stay, and incidence of complications was observed in both groups $(\mathrm{P}>0.05)$ (Table 2).

\section{Comparison of mid-term and long-term efficacy of both groups}

The 2-year survival rate and the incidence of complications after discharge of the TEVAR group was significantly higher and lower than that of the control group $(\mathrm{P}<0.05)$, respectively. No significant difference in the 1-year survival rate and the second intervention rate was observed in both groups $(\mathrm{P}>0.05)$ (Table 3$)$.

\section{Discussion and Conclusion}

$\mathrm{AD}$ is one of the most dangerous forms of vascular disease, characterized by endometrial rupture and intramural hematoma formation. Generally, the pathological process is complicated and closely related to the infiltration of inflammatory cells into the aortic wall and apoptosis of vascular smooth muscle cells [7]. Inflammation is closely related to the prognosis of AD patients [8]. The suppression of inflammation could play significant role in reducing the incidence of complications in $\mathrm{AD}$ patients and improving the prognosis. The inflammatory cytokines (IL-6, IL-8 and TNF- $\alpha$ ), released in the process of inflammation play important roles in the occurrence and development of CVD, such as myocardial infarction7. IL-6 can induce hepatocytes to synthesize high-sensitivity C-reactive protein and regulate macrophages to secrete TNF- $\alpha$, which plays a key role in vascular injury and tissue trauma [9]. TNF- $\alpha$ is an early inflammatory factor released after AD. It can inhibit the proliferation of vascular cells, promote neutrophil phagocytosis, and damage vascular endothelial cells [10]. Previous studies reported that the plasma levels of IL- 6 and TNF- $\alpha$ in AD patients were remarkably higher than those in healthy subjects [11,12]. Thus, monitoring the levels of IL- 6 and TNF- $\alpha$ is helpful for clinicians to predict prognosis, and lower levels of IL-6 and TNF- $\alpha$ are beneficial to improve the prognosis in AD patients. The present study found that the levels of IL- 6 , IL- 8 , and TNF- $\alpha$ in both groups were significantly lower than those in the control group, whereas the levels of IL-6, IL-8, and TNF- $\alpha$ in the TEVAR group were significantly lower than those in the control group (Figure 1). One potential explanation for an occurrence of inflammation in $\mathrm{AD}$ is the involvement of systemic inflammatory response syndrome (SIRS), a clinical syndrome of dysregulated inflammation that includes a massive and uncontrolled release of pro-inflammatory mediators. The causes of SIRS are believed to be exogenous substances derived from pathogenic microorganisms, such as bacterial endotoxin, and endogenous substances that contribute to inflammation, such as those released by tissue damage. TEVAR seems to decrease the postoperative stress by offering less extensive incisions, dissection, and tissue manipulation. However, these beneficial effects may be offset by the release of cytokines during intra-luminal manipulation of the thrombus using catheters in endovascular repair, resulting in SIRS24. Therefore, our result suggests that TEVAR can significantly alleviate inflammation and improve the prognosis of patients.

The alternations of immune cells were closely related to the occurrence and development of $\mathrm{AD}$ [13]. Neutrophils have chemotaxis, phagocytosis, and bactericidal lamp functions that can release IL-6 and matrix metalloprotein-9 [14]. Del Porto, et al. [15] found that the total number of WBC and neutrophils in $\mathrm{AD}$ patients was significantly higher than that in healthy people. The studies have found that the mortality risk of $\mathrm{AD}$ patients in high WBC group was significantly higher than that in the low WBC group. Moreover, the ratio of neutrophil to lymphocyte is related to the prognosis of $\mathrm{AD}$ 

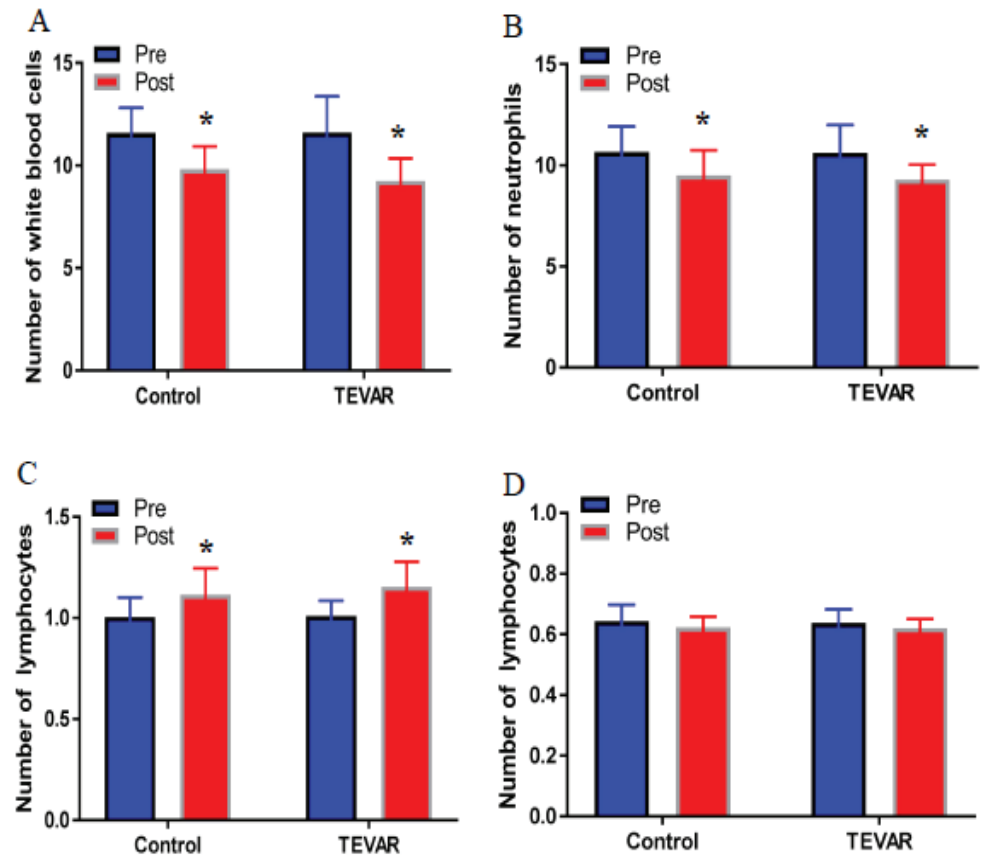

Figure 2: TEVAR decreased the expression in immune cells.

The number of WBC (A), neutrophils (B), lymphocytes (C), and monocytes (D) were measured by using a hematology analyzer. Data were expressed as mean $\pm S D(n=30)$. Fisher's protected least significant difference test was performed to analyze the differences between the control and TEVAR groups. ${ }^{*} \mathrm{P}<0.05$ compared with that pre treatment.

Table 1: Basic characteristics of patients

\begin{tabular}{|c|c|c|c|c|}
\hline Parameter & Control group $(n=30)$ & TEVAR group $(n=30)$ & $\chi^{2} / t$ & $\boldsymbol{P}$ \\
\hline Gender (male/female) & $17 / 13$ & $16 / 14$ & 0.067 & 1.000 \\
\hline Age (years) & $53.95 \pm 5.65$ & $51.89 \pm 5.38$ & 1.456 & 0.153 \\
\hline Hypertension & 23 & 21 & 0.341 & 0.771 \\
\hline Smoking & 12 & 13 & 0.069 & 1.000 \\
\hline Diabetes mellites & 4 & 3 & 0.162 & 1.000 \\
\hline Onset time (d) & $7.25 \pm 0.81$ & $7.41 \pm 0.79$ & 0.774 & 0.442 \\
\hline Pseudo thrombosis & & & 1.017 & 1.000 \\
\hline Thrombosis & 1 & 0 & & \\
\hline False lumen patency & 29 & 30 & & \\
\hline
\end{tabular}

Table 2: Comparison of curative effect of two groups of patients.

\begin{tabular}{|l|c|c|c|}
\hline \multicolumn{1}{|c|}{ Parameter } & Control group (n=30) & TEVAR group (n=30) & $\boldsymbol{P}$ \\
\hline Effective rate & $22(73.33 \%)$ & $29(96.67 \%)$ & 6.405 \\
\hline In-hospital mortality rate & $3(10.00 \%)$ & $2(6.67 \%)$ & 0.218 \\
\hline Length of hospital stay (d) & $23.12 \pm 2.36$ & $23.54 \pm 2.41$ & 0.682 \\
\hline Complications in hospital & $4(13.33 \%)$ & 0 & 0.498 \\
\hline
\end{tabular}


Table 3: Comparison of mid-term and long-term efficacy of patients.

\begin{tabular}{|c|c|c|c|c|}
\hline Parameter & Control group $(n=30)$ & TEVAR group $(n=30)$ & $\chi^{2} / t$ & $\boldsymbol{P}$ \\
\hline 1-year survival rate & $25(83.33 \%)$ & 27 (90.00\%) & 0.417 & 0.748 \\
\hline 2-year survival rate & $18(60.00 \%)$ & $26(86.67 \%)$ & 5.455 & 0.039 \\
\hline Complications after discharge & $9(30.00 \%)$ & $2(6.67 \%)$ & 5.445 & 0.042 \\
\hline Secondary intervention rate & $7(23.33 \%)$ & $1(3.33 \%)$ & 5.192 & 0.052 \\
\hline
\end{tabular}

patients, and the ratio of neutrophil to lymphocyte in the death group is higher than that in the survival group [16,17]. In this study, we measured the levels of immune cells of patients before and after the treatment. The results showed that the number of WBC and neutrophils and the number of lymphocytes in both groups significantly decreased and increased, respectively. The number of WBC and neutrophils in the TEVAR group was significantly lower than that in the control group, and the number of lymphocytes was significantly higher than that in the control group (Figure 2). The destruction of the middle layer of the vascular wall delays the formation of early $\mathrm{AD}$ lesions and aortic aneurysms, subsequently reduces the number of $\mathrm{WBC}$ and neutrophils, and increases the number of lymphocytes [18].

Previous studies reported that conservative treatment has adverse prognosis because approximately $30 \%-40 \%$ of $\mathrm{AD}$ patients can develop complex $\mathrm{AD}$ or aneurysmal degeneration [19]. The hospital mortality, complication rate, and secondary intervention rate in 30 days are high, but the 5-year survival rate is low [20]. The present study showed that the technical success rate of the TEVAR-treated group was high; its hospital mortality rate was low; the occurrence of severe complications, such as retrograde type-A dissection, and spinal cord ischemia, was low; and the short-term effect was satisfactory. Previous logistic regression analysis studies showed that age over 75 was an independent risk factor for early adverse events after surgery [21]. The results of this study showed that the effective rate of the TEVAR group was significantly higher than that of the control group. The mortality rate, length of stay, and incidence of complications in the TEVAR group were lower than those in the control group, but no statistical difference was observed in both groups (Table 2). Some studies also found that the 5-year, 10-year, and 14-year survival rates of AD patients after TEVAR treatment were $96.6 \%, 84.3 \%$, and $67.8 \%$, respectively, which were significantly higher than the long-term survival rate of the best drug conservative treatment [22]. Nienaber, et al. [23-25] found that the 5-year all-cause mortality of TEVAR group was $11.1 \%$ and $19.3 \%$, and the aorta-related mortality was $6.9 \%$ and $19.3 \%$, which were significantly lower than that of the drug treatment group. In this study, the 2-year survival rate and the incidence of complications after discharge of the TEVAR group was significantly higher and lower than those of the control group, respectively (Table 3 ).

In conclusion, TEVAR has good therapeutic effect that can greatly inhibit inflammation and improve survival rate to a certain extent. The small number of cases and no randomized controlled treatment are the limitations of present study. Therefore, a large sample of randomized controlled studies is still needed to investigate the therapeutic effect of TEVAR.

\section{Acknowledgments}

We thank Dr. Kyosuke Takeshita for carefully reading and applying changes to our manuscript.

\section{Funding and Disclosure}

This work, which was led by Zonggang Zhang and Lianjun Huang, was supported by the National Key R\&D Program of China (Grant no. 2017YFC1308000). No duality of interest during the writing of this manuscript was declared by the authors.

\section{Contribution Statement}

MA, MY, WMZ, and ZZZ contributed to the conceptualization and formulation of the experimental design and the interpretation of the experimental results. MA, MY, WMZ, and AA performed the experiments and analyzed the data.MA, MY, and WMZ wrote the manuscript. MA, ZZZ, HJZ, and LJH ensured the integrity of the entire work. This manuscript was revised by its authors, all of whom decided to submit this version for publication.

\section{References}

1. Silaschi M, Byrne J, Wendler O (2015) Aortic dissection: medical, interventional and surgical management. Heart 103: 78-87.

2. Bicknell C, Powell JT (2014) Aortic disease: thoracic endovascular aortic repair. Heart 101: 586-591.

3. Ehsan O, Choudry UK, Rana A, Khan ZA (2018) Endovascular Aortic Repair for Thoracic Aortic Injuries. J Coll Physicians Surg Pak 28: S178-S180.

4. Muehle A, Shah A, Khoynezhad A (2015) Thoracic Endovascular Aortic Repair in the Ascending Aorta. Innovations (Phila) 10: 363367.

5. Wu Z, Zheng X, Fang X, He Y, Li D, et al. (2017) Endovascular Management of Native Postcoarctation Thoracic Aortic Aneurysms. Cardiovasc Intervent Radiol 40: 1529-1534.

6. Yisireyili $M$, Uchida $Y$, Yamamoto $K$, Nakayama T, Cheng XW, et al. (2018) Angiotensin receptor blocker irbesartan reduces stressinduced intestinal inflammation via AT1a signaling and ACE2dependent mechanism in mice. Brain Behav Immun 69: 167-179.

7. Zeng T, Shi L, Ji Q, Shi Y, Huang Y, et al. (2018) Cytokines in aortic dissection. Clin Chim Acta 486: 177-182.

8. Cifani N, Proietta M, Tritapepe L, Di Gioia C, Ferri L, et al. (2015) Stanford-A acute aortic dissection, inflammation, and metalloproteinases: a review. Ann Med 47: 441-446.

9. Kokje VBC, Gäbel G, Koole D, Northoff BH, Holdt LM, et al. (2016) IL-6: A Janus-like factor in abdominal aortic aneurysm disease. Atherosclerosis 251: 139-146.

10. Prisco AR, Hoffmann BR, Kaczorowski CC, McDermott-Roe C, Stodola TJ, et al. (2016) Tumor Necrosis Factor $\alpha$ Regulates Endothelial Progenitor Cell Migration via CADM1 and NF-kB. Stem Cells 34: 1922-1933.

11. Ijaz T, Tilton RG, Brasier AR (2016) Cytokine amplification and macrophage effector functions in aortic inflammation and abdominal aortic aneurysm formation. J Thorac Dis 8: E746-E754. 
12. Moutafi AC, Alissafi T, Chamakou A, Chryssanthopoulos S, Thanopoulos V, et al. (2012) Neurohormonal activity and vascular properties late after aortic coarctation repair. Int J Cardiol 159: 211216.

13. Visonà SD, de Boer OJ, Mackaaij C, de Boer HH, Pertiwi KR, et al. (2018) Immunophenotypic analysis of the chronological events of tissue repair in aortic medial dissections. Cardiovasc Pathol 34: 9-14.

14. Górska K, Paplińska-Goryca M, Nejman-Gryz P, Goryca K, Krenke R (2017) Eosinophilic and Neutrophilic Airway Inflammation in the Phenotyping of Mild-to-Moderate Asthma and Chronic Obstructive Pulmonary Disease. COPD 14: 181-189.

15. del Porto F, Proietta M, Tritapepe L, Miraldi F, Koverech A, et al. (2010) Inflammation and immune response in acute aortic dissection. Ann Med 42: 622-629.

16. Oz K, lyigun T, Karaman Z, Çelik Ö, Akbay E, et al. (2017) Prognostic Value of Neutrophil to Lymphocyte Ratio and Risk Factors for Mortality in Patients with Stanford Type A Aortic Dissection. Heart Surg Forum 20: E119-E123.

17. Kalkan ME, Kalkan AK, Gündeş A, Yanartaş M, Oztürk S, et al. (2017) Neutrophil to lymphocyte ratio: a novel marker for predicting hospital mortality of patients with acute type A aortic dissection. Perfusion 32: 321-327.

18. Onuk T, Güngör B, Karataş B, Ipek G, Akyüz S, et al. (2015) Increased Neutrophil to Lymphocyte Ratio is Associated with In-Hospital Mortality in Patients with Aortic Dissection. Clin Lab 61: 1275-1282.
19. Tang $Y$, Liao Z, Han L, Tang H, Song Z, et al. (2017) Long-term results of modified sandwich repair of aortic root in 151 patients with acute type A aortic dissection. Interact Cardiovasc Thorac Surg 25: 109113.

20. Kamohara K, Koga S, Takaki J, Yoshida N, Furukawa K, et al. (2017) Long-term durability of preserved aortic root after repair of acute type A aortic dissection. Gen Thorac Cardiovasc Surg 65: 441-448.

21. Omura A, Matsuda H, Minami H, Nakai H, Henmi S, et al. (2017) Early and Late Outcomes of Operation for Acute Type A Aortic Dissection in Patients Aged 80 Years and Older. Ann Thorac Surg 103: 131-138.

22. Merkle J, Sabashnikov A, Weber C, Schlachtenberger G, Maier J, et al. (2018) Impact of age on early outcomes and long-term survival of patients undergoing aortic repair with Stanford A dissection. Perfusion 33: 687-695.

23. Nienaber $C A$, Kische $S$, Rousseau $H$, Eggebrecht $H$, Rehders TC, et al. (2013) Endovascular repair of type B aortic dissection: longterm results of the randomized investigation of stent grafts in aortic dissection trial. Circ Cardiovasc Interv 6: 407-416.

24. Tsilimigras DI, Sigala F, Karaolanis G, Ntanasis-Stathopoulos I, Spartalis E, et al. (2018) Cytokines as biomarkers of inflammatory response after open versus endovascular repair of abdominal aortic aneurysms: a systematic review. Acta Pharmacol Sin 39: 1164-1175.

25. Golia E, Limongelli G, Natale F, Fimiani F, Maddaloni V, et al. (2014) Inflammation and cardiovascular disease: from pathogenesis to therapeutic target. Curr Atheroscler Rep 16: 435. 